\title{
Medicamentos psicotrópicos para idosos: Quais especialidades médicas mais prescrevem?
}

\author{
Psychotropic drugs for the elderly: Which medical specialties prescribe the most? \\ Fármacos psicotrópicos para personas mayores: ¿Qué especialidades médicas prescriben más?
}

Rafaela Fernandes Lourenço ORCID: https://orcid.org/0000-0003-4738-3486 Universidade Federal de São João del Rei, Brasil E-mail: rafaelaflourenco@gmail.com

Maria Luiza Gama Costa ORCID:https://orcid.org/0000-0001-7469-9852 Universidade Federal de São João del Rei, Brasil E-mail: marialgama.mlg@gmail.com

Anna Karolyna Rodrigues Cunha ORCID: https://orcid.org/0000-0002-2370-5682 Universidade Federal de São João del Rei, Brasil E-mail: karolynarc@gmail.com

Juliana Souza Martins ORCID: https://orcid.org/0000-0001-7800-9193 Universidade Federal de São João del Rei, Brasil

E-mail: juszmartins@gmail.com

Rafaela Candian Filgueiras Silva ORCID: https://orcid.org/0000-0002-2230-4035 Universidade Federal de São João del Rei, Brasil

E-mail: rafaelacandian@gmail.com

Pedro Henrique Ferreira ORCID: https://orcid.org/0000-0003-1721-3275 Universidade Federal de São João del Rei, Brasil E-mail: pedrohferreira25@gmail.com

Adam Nascimento Silva

ORCID: https://orcid.org/0000-0001-6224-0171 Universidade Federal de São João del Rei, Brasil

E-mail: nascimento-adam@ hotmail.com

Thays Santos Mendonça

ORCID: https://orcid.org/0000-0002-7005-8780 Universidade Federal de São João del Rei, Brasil

E-mail: thayssm.farmacia@gmail.com

André Oliveira Baldoni

ORCID: https://orcid.org/0000-0001-6379-0415 Universidade Federal de São João del Rei, Brasil

E-mail: andrebaldoni@ufsj.edu.br

Denise Alves Guimarães

ORCID: https://orcid.org/0000-0002-3539-6733 Universidade Federal de São João del Rei, Brasil

E-mail: alvesguimaraesdenise@gmail.com

\begin{abstract}
Resumo
Esse estudo teve o objetivo de analisar o perfil das especialidades médicas dos prescritores de psicotrópicos para idosos atendidos pelo Sistema Único de Saúde (SUS) de um município mineiro de médio porte. Trata-se de um estudo descritivo realizado a partir de dados secundários de dispensação de medicamentos registrados no Sistema Informatizado de Saúde entre janeiro e dezembro de 2017. Para esta análise foi considerada a especialidade de contratação dos médicos prescritores informada pela Secretaria Municipal de Saúde do município estudado. Foram realizadas 23.599 dispensações para 4.833 idosos, solicitadas por 194 médicos prescritores. O número de prescrições por especialidade foi maior entre médicos Clínicos Gerais - 8.463 (35,9\%), Médicos da Estratégia da Saúde da Família - $6.927(29,4 \%)$ e Psiquiatras - $5.152(21,8 \%)$. Analisando a média de prescrições por profissional de cada especialidade, foi observado que Psiquiatras são os que mais prescrevem, seguidos pelos Mastologistas e pelos Cirurgiões Gerais, com médias de 1.717, 957 e 215 prescrições por profissional, respectivamente. A média geral é de 122 prescrições por especialista. Concluiu-se que a elevada frequência de prescrições de medicamentos psicotrópicos aos idosos por médicos que não possuem formação em psiquiatria e/ou geriatria não é infração legal ou ética, no
\end{abstract}


entanto, considerando a complexidade do cuidado ao idoso e as particularidades desses medicamentos, desde a escolha da farmacoterapia até o momento da desprescrição, faz-se necessária a reflexão a respeito dos desafios vindouros, sobretudo no cenário de rápido processo de envelhecimento populacional brasileiro e da escassez de médicos geriatras.

Palavras-chave: Prescrições; Psicotrópico; Idoso; Sistema Único de Saúde.

\begin{abstract}
This study aimed to analyze the profile of the medical specialties of psychotropic prescribers for the elderly assisted by the Unified Health System (SUS) in a medium-sized city in Minas Gerais. This is a descriptive study carried out using secondary data on drug dispensing registered in the Computerized Health System between January and December 2017. For this analysis, the specialty of hiring prescribing doctors informed by the Municipal Health Secretariat was considered. 23.599 dispensations were made for 4.833 elderlies, requested by 194 prescribing doctors. The number of prescriptions by specialty was higher among General Physicians - 8.463 (35.9\%), Family Health Strategy Physicians - 6.927 (29.4\%) and Psychiatrists - 5.152 (21.8\%). Analyzing the average number of prescriptions per professional in each specialty, it was observed that Psychiatrists are the ones who prescribe the most, followed by the Mastologists and by General Surgeons, with averages of 1.717, 957 and 215 prescriptions per professional, respectively. The overall average is 122 prescriptions per specialist. It was concluded that the high frequency of psychotropic medications prescriptions to the elderly by doctors who do not have graduation in psychiatry and/or geriatrics is not a legal or ethical infraction, however, considering the care complexity for the elderly and these medications particularities, from the pharmacotherapy choice to the moment of medication interruption, it is necessary to reflect on the challenges ahead, especially in the scenario of fast Brazilian population aging process and the shortage of geriatric doctors.
\end{abstract}

Keywords: Prescriptions; Psychotropic drugs; Elderly; Public health.

\title{
Resumen
}

Este estudio tuvo como objetivo analizar el perfil de las especialidades médicas de los prescriptores de medicamentos para personas mayors atendidas por el Sistema Único de Salud (SUS) en una ciudad mediana de Minas Gerais. Se trata de un estudio descriptivo con base en datos secundarios de dispensación de medicamentos registrados en el Sistema Informático de Salud (SIS) de enero a diciembre de 2017. Se consideró la especialidad de contratación de médicos prescriptores informados por la Secretaría Municipal de Salud del municipio. Hubo 23.599 dispensaciones para 4.833 ancianos, solicitadas por 194 médicos prescriptores. El número de prescripciones por especialidad fue más significativo entre los médicos generalistas - 8.463 (35,9\%), seguido por los medicos del programa "Estrategia de Salud Familiar" - $6.927(29,4 \%)$ y los psiquiatras - $5.152(21,8 \%)$. Una vez analizado el promedio de prescripciones por profesional en cada especialidad, los psiquiatras son los que más prescriben: 1.717 prescripciones por profesional, seguidos de los mastólogos con 957 y los cirujanos generales con 215 . El promedio general es de 122 recetas por especialista. Se concluyó que la alta frecuencia de prescripción de medicamentos a ancianos por parte de médicos sin formación en psiquiatría y/o geriatría no es una infracción legal ni ética. Sin embargo, considerando la complejidad de la atención a las personas mayores y las particularidades de estos fármacos, es necesario reflecionar acerca de los desafíos venideros, en el rápido envejecimiento de la población brasileña y escasez de geriatras.

Palabras clave: Recetas; Medicamento; Personas mayores; Sistema Único de Salud.

\section{Introdução}

Para a população assistida pela Atenção Primária em Saúde (APS) no Sistema Único de Saúde (SUS) a polifarmácia é uma realidade que merece atenção. Com o aumento da expectativa de vida e, consequentemente, das condições crônicas em saúde, o uso de múltiplos fármacos tem se tornado mais frequente ao longo do tempo (Nascimento et al., 2017). Um estudo nacional demonstra que o uso de medicamentos cresce conforme aumenta a faixa etária, chegando a 92,1\% em pessoas com 65 anos de idade ou mais (Costa et al., 2017). Tal realidade, aliada ao rápido processo de envelhecimento populacional brasileiro, evidencia a necessidade de estudos que analisem aspectos sobre racionalidade de uso dos medicamentos (Instituto Brasieiro de Geografia e estatística [IBGE], 2018).

Dentre os medicamentos utilizados pelos idosos, muitos são considerados potencialmente inapropriados, dentre eles alguns psicotrópicos (American Geriatrics Society, 2019), que são os medicamentos que atuam sobre o Sistema Nervoso Central (SNC) modificando o seufuncionamento, podendo provocar alterações no humor, nas percepções, nos comportamentos e nos estados da consciência ou funções psíquicas (Edwards et al., 1981). Os antidepressivos e os benzodiazepínicos são os 
psicotrópicos mais consumidos no mundo (Ćurković et al, 2016) e o seu uso crônico e indiscriminado tornou-se cada vez mais comum (Mezzari \& Moehlecke, 2015).

Nas últimas duas décadas tem sido registrado o aumento das prescrições desta classe de medicamentos para a população brasileira, especialmente na APS e para pacientes com condições crônicas, culminando no seu uso abusivo; com o agravante de que muitos usuários não possuem condições clínicas que justifiquem essa prescrição ou não são adequadamente avaliados quanto à necessidade de prescrição (Rosa et al., 2012; de Moura et al., 2016).

Outro fator que pode contribuir para o problema do uso indiscriminado de psicotrópicos é o fato de que a dispensação desses medicamentos frequentemente ocorre por solicitação dos pacientes que já os utilizam, sem reavaliação adequada do prescritor; bem como o fato de que, na maioria das vezes, a última prescrição dos psicotrópicos não foi realizada por médicos psiquiatras (Rosa et al., 2012). Um estudo realizado em Unidades Básicas de Saúde da Paraíba aponta, ainda, o receio dos pacientes em relação à desprescrição dos psicotrópicos, o que pode estar relacionado a um conjunto de fatores, dentre os quais a dependência física e psicológica causada por alguns deles (Sarmento \& Santos, 2020). Além disso, os medicamentos constituem parte do tratamento e não possibilitam, isoladamente, a solução ou cura de doenças; questão que impõe aos profissionais de saúde a discussão sobre a necessidade de prescrição consciente, orientação direcionada ao usuário quanto à autoadministração e acompanhamento na rede de saúde (Ministério da Saúde, 2019).

O uso inapropriado de psicotrópicos está associado a um maior risco de lesões por queda e a hospitalizações de idosos (Yu et al., 2017). Além disso, essa classe de medicamentos aumenta o risco de delirium e pode causar dependência física e psicológica dos usuários crônicos (Oliveira \& Santos, 2016).

Para que seja possível evitar o uso irracional e por tempo indeterminado desses medicamentos, torna-se, portanto, imperativo a realização de estudos que subsidiem intervenções para racionalizar a prescrição, a dispensação e o uso destes medicamentos para idosos (Barbiani at al., 2014).

Nesse sentido, este estudo objetiva analisar o perfil das especialidades dos prescritores de psicotrópicos para idosos atendidos pelo Sistema Único de Saúde (SUS).

\section{Metodologia}

Considerando a complexidade e a interdisciplinaridade características do campo da Saúde, realizou-se estudo qualitativo, de caráter exploratório (Minayo, 2014; Minayo \& Deslandes \& Cruz Neto, 2015) a partir da análise descritiva de dados secundários, utilizando o registro de dispensação de medicamentos pelo Sistema Informatizado de Saúde (SIS) de um município do Estado de Minas Gerais, com população estimada em 240.000 habitantes (IBGE, n.d.).

O SIS consta informações referentes à todas as dispensações de medicamentos no âmbito do SUS, das cinco farmácias do componente básico da Assistência Farmacêutica do município, o que permitiu a inclusão de todos os dados referentes à dispensação de psicotrópicos para idosos, com 60 anos ou mais, durante o ano de 2017. Essas informações foram compiladas em um banco de dados construído pelos pesquisadores e foram analisados os seguintes parâmetros: especialidade de contratação pelo município dos profissionais médicos que prescreveram psicotrópicos, números absolutos de prescrições e a média de prescrição por profissional de cada especialidade. Foram excluídos os médicos cuja especialidade não foi informada pela Secretaria Municipal de Saúde. Utilizou-se o Microsoft Excel ${ }^{\circledR}$ para a análise dos dados.

Foram considerados todos os psicotrópicos presentes na Relação Municipal de Medicamentos Essenciais (REMUME): Ácido Valpróico, Alprazolam, Amitriptilina, Biperideno, Carbamazepina, Carbonato de Lítio, Clomipramina, Clonazepam, Clorpromazina, Diazepam, Fenitoina, Fenobarbital, Fluoxetina, Flurazepam, Haloperidol, Imipramina, Levomepromazina, Nitrazepan, Nortriptilina, Oxcarbazepina, Sertralina, Tioridazina e Topiramato. 
O estudo foi aprovado pelo comitê de ética em pesquisa pelo parecer 3.053.191 e possuiu suporte financeiro apoio da Coordenação de Aperfeiçoamento de Pessoal de Nível Superior - Brasil (CAPES) - Código de Financiamento 001.

\section{Resultados}

Durante o período analisado, foram realizadas 23.599 dispensações de medicamentos psicotrópicos para um total de 4833 idosos, solicitadas pelo conjunto de 194 médicos prescritores, o que corresponde a 23 especialidades distintas contratadas pelo município (Tabela1).

O número absoluto de prescrições por especialidade foi maior entre os médicos Clínicos Gerais, com 8.463 prescrições (35,9\%), seguido pelos Médicos da Estratégia da Saúde da Família, com 6927 prescrições (29,4\%), os quais correspondem a $65,1 \%$ do total. Os psiquiatras realizaram $5.152(21,8 \%)$ prescrições.

No entanto, analisando a média de prescrições por profissional de cada especialidade, psiquiatras prescreveram 14 vezes mais que a média geral (1.717 prescrições por médico psiquiatra, enquanto a média geral é de 122 prescrições por médico), sendo que esses especialistas representam apenas 1,54\% do total de prescritores, com apenas três médicos registrados. Além disso, 957 dispensações de psicotrópicos para idosos foram realizadas para atender as prescrições de um médico mastologista e 268 dispensações foram provenientes de prescrições feitas por 10 pediatras.

Tabela 1 - Especialidade dos médicos prescritores de psicotrópicos para idosos ( $\mathrm{n}$ = 194).

\begin{tabular}{lccc}
\hline $\begin{array}{l}\text { Especialidade de } \\
\text { Contratação }\end{array}$ & $\begin{array}{l}\text { Número de } \\
\text { Médicos }\end{array}$ & $\begin{array}{l}\text { Número de } \\
\text { Prescrições }\end{array}$ & $\begin{array}{l}\text { Média de } \\
\text { Prescrição por } \\
\text { Médico }\end{array}$ \\
\hline Psiquiatria & 3 & 5152 & 1717 \\
Mastologia & 1 & 957 & 957 \\
Cirurgia Geral & 5 & 1076 & 215 \\
Reumatologia & 2 & 339 & 170 \\
Clínica Geral & 53 & 8463 & 160 \\
Médico da Estratégia de Saúde da & 60 & 6927 & 115 \\
Família & & & 31 \\
Medicina do Trabalho & 2 & 62 & 28 \\
Nefrologia & 1 & 28 & 27 \\
Pediatria & 10 & 268 & 22 \\
Gastroenterologia & 2 & 43 & 19 \\
Neurologia & 3 & 56 & 5 \\
Endocrinologia e Metabologia & 4 & 75 & 28 \\
Coloproctologia & 1 & 5 & \\
Outros ${ }^{1}$ & 47 & 148 & \\
\hline
\end{tabular}

${ }^{1}$ Radiologia e Diagnóstico por Imagem, Cardiologista, Dermatologista, Ginecologista e Obstetra, Generalista, Urologista, Infectologista, Ortopedista e Traumatologista, Hematologista e Neurocirurgião. Fonte: Autores (2021). 


\section{Discussão}

Os resultados deste estudo apontam que $86,9 \%$ das prescrições foram realizadas por três especialidades, enquanto as outras 20 especialidades foram responsáveis por 13,1\% das demais prescrições. Na literatura nacional não há estudos que analisaram o perfil de prescritores de psicotrópicos especificamente para idosos. Foram identificados apenas estudos brasileiros que avaliaram as especialidades médicas que mais prescrevem psicotrópicos para a população geral. Dentre esses, um estudo mineiro publicado em 2019, evidenciou que, no biênio 2015 - 2016, a maioria dos psicotrópicos dispensados na farmácia básica do município investigado havia sido prescrita por Clínicos Gerais, seguidos por Psiquiatras, Neurologistas e Cardiologistas (Santos et al., 2019). De modo semelhante, um estudo realizado no Ceará demonstrou que as principais especialidades envolvidas na dispensação de psicotrópicos para a população geral foram os Clínicos Gerais (72,92\% e 81,59\% no primeiro e segundo períodos de coleta, respectivamente) e os Psiquiatras (23,69\% e 10,45\%, respectivamente). As outras especialidades foram responsáveis por 3,38\% e 7,96\% das prescrições, no primeiro e segundo períodos de coleta, respectivamente (Reis, Matos, \& Melo, 2017). Isso posto, observa-se que, de forma geral, os resultados encontrados neste estudo, bem como o perfil de especialidades médicas que mais prescrevem psicotrópicos seguem as tendências nacionais apontadas na literatura científica para a população geral. Isso evidencia a necessidade de investimento em pesquisas na área, bem como a revisão e implementação de políticas e serviços de saúde no país que atendam e conheçam as particularidades da população geriátrica brasileira.

O elevado número de prescrições realizadas por apenas três psiquiatras está associado à realidade dos municípios de pequeno e médio porte, nos quais essa especialidade médica é pouco oferecida pela rede pública, com consequente sobrecarga do sistema de saúde e dos profissionais disponíveis, bem como em prejuízos na integralidade da atenção às especificidades do idoso. Pode-se inferir que a dificuldade de acesso ao especialista em saúde mental possui impacto na forma como o conjunto de especialidades médicas manejam a clínica do idoso, a qual mostra-se prioritariamente direcionada à prescrição de medicamentos a partir do modelo biomédico em detrimento do cuidado centrado na pessoa (Wenceslau \& Ortega, 2015).

Quanto ao elevado número de prescrições de psicotrópicos realizadas por mastologistas, sabe-se que os pacientes encaminhados a esse especialista geralmente têm diagnóstico de câncer de mama e, em comparação com a população geral, possuem risco aumentado de sintomas e transtornos depressivos persistentes, pois o diagnóstico de câncer de mama associa-se a alterações negativas na imagem corporal, sexualidade e relações interpessoais, além do próprio estigma social, sendo considerado o tipo de câncer mais temido pelas mulheres (Ferreira et al., 2015). Desse modo, pode-se inferir que a consulta com o mastologista se revela um momento oportuno para a prescrição e/ou renovação da receita de psicotrópicos (Ferreira et al., 2015; Pereira et al., 2020).

Ademais, sabe-se que a depressão também está relacionada à baixa adesão ao tratamento oncológico. Assim, o uso de medicamentos psicotrópicos, além de aliviar os sintomas depressivos, favorece a adesão do paciente ao tratamento contra o câncer, tendo como efeitos adicionais a redução de sintomas como náuseas, vômitos, dor e fadiga frequentemente associados à quimioterapia (Bottino et al., 2009).

Em relação à prescrição de psicotrópicos para idosos realizada por pediatras, propõe-se a hipótese de que os idosos muitas vezes renunciam ao autocuidado em detrimento do cuidado dos netos, já que o modelo de família mosaico presente em muitos lares brasileiros, associado à dificuldade de agendamento de consultas pelos idosos, favorece a utilização da consulta das progênies com o pediatra para renovação de suas próprias receitas (Cavenaghi \& Alves, 2018).

Analisando o conjunto e o perfil de prescritores do presente estudo, vale salientar a importância das discussões apontadas na literatura a respeito da complexidade da prescrição de psicotrópicos e, ainda, o fato de os pacientes estarem mais propensos a não terem um real diagnóstico psiquiátrico que justifique a prescrição médica. Um grande consumo de psicotrópicos pode ocasionar déficit cognitivo, síncope, quedas e fraturas nesse grupo. Ainda pode haver o uso concomitante 
de outros medicamentos pelos idosos, estabelecendo a condição de polifarmácia, com piora do estado de saúde mental e levando o idoso a ser medicado com fármacos voltados aos aspectos psicológicos e comportamentais (de Moura et al., 2016).

Em um estudo brasileiro sobre o acompanhamento e uso de psicotrópicos na APS para a população geral de um município paraibano, percebeu-se que a inexistência de acompanhamento ( $74 \%$ dos usuários) é sugestiva de que o motivo dos atendimentos na Unidade Básica de Saúde seja para renovação de receita. Ainda nesse estudo, os médicos generalistas foram os maiores prescritores de psicotrópicos e demonstraram uma deficiência de capacitação destes para um atendimento qualificado na APS. Logo, para evitar o uso indiscriminado desses medicamentos, viu-se a necessidade de capacitar esses profissionais e utilizar o matriciamento no acompanhamento desses usuários (Sarmento \& Santos, 2019).

A prescrição de medicamentos para idosos requer planejamento e monitoramento para se evitar a polifarmácia não qualificada e consequentemente a cascata iatrogênica, portanto, a revisão dos medicamentos deve ser realizada em toda consulta de pacientes idosos (Moraes, 2012). Sendo assim, é importante que os médicos acompanhem e reavaliem os pacientes em uso de psicotrópicos a cada dois meses (Sarmento e Santos, 2019).

Estudo de 2008 propôs um novo modelo de cuidado ao idoso que coaduna com os objetivos propostos por esse estudo, abordando a construção de um fluxo bem desenhado de ações de educação, promoção de saúde, iniciando a linha de cuidados no acolhimento e no monitoramento do idoso e se encerrando somente nos momentos finais da vida, comos cuidados paliativos, fortalecendo o foco no indivíduo e em suas particularidades, acrescentando não só para a qualidade de vida do idoso mas também para a sustentabilidade do sistema de saúde brasileiro (Veras e Oliveira, 2018).

Diante do cenário, o processo da desprescrição racional e sistematizado de psicotrópicos, o qual envolve a redução da dose ou interrupção no uso de um medicamento, deve ser amplamente discutido entre as especialidades médicas e presente na formação acadêmica. Devem ser consideradas nesse processo a opção pela desprescrição, a experiência do médico prescritor, a aceitabilidade do paciente e a organização dos serviços de saúde, tendo como objetivo, auxiliar o médico a reduzir danos, os custos em saúde e melhorar a qualidade de vida do idoso (NHS, 2019).

Em relação à limitação do estudo, é importante destacar que a especialidade de contratação dos médicos foi fornecida pelo sistema de dados secundários do município, podendo apresentar problemas de informações e nem sempre correspondendo à área de atuação real do profissional. Isso impede uma correlação direta entre a prescrição e a especialidade, contudo, a potencialidade do estudo se fundamenta na característica inovadora e temática relevante do trabalho, que evidencia qual é o perfil dos prescritores de psicotrópicos para toda a população geriátrica do município que possui a $12^{\circ}$ maior população do estado de Minas Gerais.

\section{Conclusão}

O panorama de dispensações de psicotrópicos para idosos no município aponta problemas relacionados à organização da assistência à população geriátrica. Diante da diversidade e da falta de coerência entre a especialidade médica responsável pelo atendimento, o perfil da população atendida e dos medicamentos prescritos, torna-se imperativo a implementação de estratégias que contribuam para a racionalização no uso dos medicamentos, tais como: fortalecimento da Educação Permanente em Saúde, Matriciamento e integração entre equipes da APS e equipes especializadas em geriatria e saúde mental, para que os médicos da rede considerem a complexidade do cuidado ao idoso nos serviços públicos de saúde. Tais questões são fundamentais para o avanço das discussões das especificidades de prescrição para idosos e a importância da avaliação clínica. Dessa forma, é possível evitar a prática de renovação de receitas ao promover a implementação de protocolos de desprescrição de medicamentos, incluindo psicotrópicos, para a população idosa.

Nesse sentido, sugerem-se trabalhos futuros que possam elucidar, com maior profundidade, os múltiplos fatores associados ao manejo dos cuidados aos idosos, à formação e atuação médica frente à saúde mental desse grupo de pacientes, 
especialmente relacionados à prescrição e uso de psicofármacos, elucidando riscos e benefícios envolvidos. Além disso, podem ser melhor evidenciados e discutidos os contextos do processo de saúde da pessoa idosa, cujas demandas de cuidados perpassam diferentes especialidades médicas, com a possibilidade estudar mais profundamente as particularidades desses atendimentos na Atenção Primária.

\section{Referências}

American Geriatrics Society 2019 Updated AGS Beers Criteria® for Potentially Inappropriate Medication Use in Older Adults. (2019). Journal of the American Geriatrics Society, 67(4), 674-694. https://doi.org/10.1111/jgs.15767

Barbiani, R., Junges, J. R., Asquidamine, F., \& Sugizaki, E. (2014). Metamorfoses da medicalização e seus impactos na família brasileira. Physis: Revista de Saúde Coletiva, 24(2), 567-587. https://doi.org/10.1590/s0103-73312014000200013

Bottino, S. M. B., Fráguas, R., \& Gattaz, W. F. (2009). Depressão e câncer. Archives of Clinical Psychiatry (São Paulo), 36(3), 109-115. https://doi.org/10.1590/s0101-60832009000900007

Cavenaghi, S., \& Alves, J. E. D. (2018). Mulheres Chefes De Família No Brasil: Avanços E Desafios (Estudos Sobre seguro, n³2 ed.) [E-book]. https://www.ens.edu.br/arquivos/mulheres-chefes-de-familia-no-brasil-estudo-sobre-seguro-edicao-32_1_2.pdf

Costa, C. M. F. N., Silveira, M. R., Acurcio, F. D. A., Guerra Junior, A. A., Guibu, I. A., Costa, K. S., Karnikowski, M. G. D. O., Soeiro, O. M., Leite, S. N., Costa, E. A., Nascimento, R. C. R., Araújo, V. E. D., \& Álvares, J. (2017). Use of medicines by patients of the primary health care of the Brazilian Unified Health System. Revista de Saúde Pública, 51, 18s. https://doi.org/10.11606/s1518-8787.2017051007144

Ćurković, M., Dodig-Ćurković, K., Petek Erić, A., Kralik, K., \& Pivac, N. (2016). Psychotropic medications in older adults: A review. Psychiatria Danubina, $28(1), 13-24$

de Moraes, E. N. (2012). Atenção à saúde do idoso: Aspectos Conceituais.. Organização Pan-Americana da Saúde. https://bibliodigital.unijui.edu.br:8443/xmlui/bitstream/handle/123456789/5574/aten\%C3\%A7\%C3\%A3o\%20a\%20saude\%20do\%20idoso.pdf?sequence=1

de Moura, D. C. N., Pinto, J. R., Martins, P., Arruda Pedrosa, K., \& Carneiro, M. G. D. (2016). Uso abusivo de psicotrópicos pela demanda da estratégia saúde da família: Revisão integrativa da literatura. SANARE - Revista de Políticas Públicas, 15(2), 136-144. https://sanare.emnuvens.com.br/sanare/article/view/1048

Edwards, G., Organización Mundial de la Salud, \& Arif, A. E. (1981). Los Problemas de la droga en el contexto sociocultural: una base para la formulación de políticas y la planificación de programas. Ginebra: Organización Mundial de la Salud.

Ferreira, A. S., Bicalho, B. P., Oda, J. M. M., Duarte, S. J. H., \& Machado, R. M. (2015). Câncer De Mama: Estimativa Da Prevalência De Ansiedade E Depressão Em Pacientes Em Tratamento Ambulatorial. Arquivos de Ciências Da Saúde Da UNIPAR, 19(3), 185-189. https://doi.org/10.25110/arqsaude.v19i3.2015.5548

Instituto Brasileiro de Geografia e Estatística. (n.d.). https://cidades.ibge.gov.br/brasil/mg/divinopolis/panorama. IBGE. https://cidades.ibge.gov.br/brasil/mg/divinopolis/panorama

Instituto Brasileiro de Geografia e Estatística. (2018, April 24). Número de idosos cresce $18 \%$ em 5 anose ultrapassa 30 milhões em 2017. https://agenciadenoticias.ibge.gov.br/agencia-noticias/2012-agencia-de-noticias/noticias/20980-numero-de-idosos-cresce-18-em-5-anos-e-ultrapassa-30milhoes-em-2017

Mezzari, R., \& Moehlecke, I. B. P. (2015). Desafios na prescrição de benzodiazepínicos em unidades básicas de saúde. Rev. AMRIGS, 59(3), 198-203. https://pesquisa.bvsalud.org/portal/resource/pt/biblio-835416

Minayo, M.C.S.(2014). O desafio do conhecimento. Pesquisa quantitativa em saúde. In O desafio do conhecimento (pp 39-53). São Paulo: Hucitec.

Minayo, M.C.S., Deslandes, S.F., Cruz Neto, O.G.R. (2015). Pesquisa social: teoria, método e criatividade. In Pesquisa social: teoria, método e criatividade (pp 31-49). Petrópolis: Vozes

Ministério da Saúde, Secretaria de Ciência, Tecnologia e Insumos Estratégicos, \& Departamento de Assistência Farmacêutica e Insumos Estratégicos. (2019). USO DE MEDICAMENTOS E MEDICALIZAÇÃO DA VIDA: Recomendações e estratégias http://bvsms.saude.gov.br/bvs/publicacoes/medica mentos_medicalizacao_recomendacoes_estrategia_led.pdf

Nascimento, R. C. R. M. D., Álvares, J., Guerra Junior, A. A., Gomes, I. C., Silveira, M. R., Costa, E. A., Leite, S. N., Costa, K. S., Soeiro, O. M., Guibu, I. A., Karnikowski, M. G. D. O., \& Acurcio, F. D. A. (2017). Polypharmacy: a challenge for the primary health care of the Brazilian Unified Health System. Revista de Saúde Pública, 51(suppl.2). https://doi.org/10.11606/s1518-8787.2017051007136

NHS. (2019). Deprescribing: A Practical Guide [E-book]. NHS Derby and Derbyshire CCG Medicines Management Team. http://www.derbyshiremedicinesmanagement.nhs.uk/assets/Clinical_Guidelines/clinical_guidelines_front_page/Deprescribing.pdf

Oliveira, L. P. B. A. D., \& Santos, S. M. A. D. (2016). An integrative review of drug utilization by the elderly in primary health care. Revista Da Escola de Enfermagem Da USP, 50(1), 163-174. https://doi.org/10.1590/s0080-623420160000100021

Pereira, A. A. C., Passarin, N. D. P., Coimbra, J. H., Pacheco, G. G., \& Rangel, M. P. (2020). Avaliação da Qualidade de Vida e Prevalência de Sintomas Depressivos em Pacientes Oncológicos Submetidos à Radioterapia. Revista Brasileira de Cancerologia, 66(1), e-12775. https://doi.org/10.32635/21769745.rbc.2020v66n1.775 
Research, Society and Development, v. 10, n. 9, e32910918075, 2021

(CC BY 4.0) | ISSN 2525-3409 | DOI: http://dx.doi.org/10.33448/rsd-v10i9.18075

Reis, A. G., Matos, M. F. S., \& Melo, O. F. (2017). Perfil de prescrições de psicotrópicos em farmácia comunitária. SANARE - Revista de Políticas Públicas, 16(2), 37-41. https://doi.org/10.36925/sanare.v16i2.1176

Rosa, F. S., Monteiro, M. T. M., Fortunato, J. J., \& Galato, D. (2012). A prescrição de psicotrópicos e a reavaliação médica. Jornal Brasileiro de Psiquiatria, 61(1), 52-53. https://doi.org/10.1590/s0047-20852012000100011

Santos, M. E. R., Neves, N. C. V., Almeida, J. C. D. S., Amparo, T. R., Piau, A. V., \& Rodrigues-das-Dôres, R. G. (2019). Consumo De Fármacos Psicotrópicos Em Uma Farmácia Básica De Congonhas, Minas Gerais, Brasil. Infarma - Ciências Farmacêuticas, 31(4), 285-292. https://doi.org/10.14450/2318-9312.v31.e4.a2019.pp285-292

Sarmento, G. A., \& Santos, S. D. (2020). Perspectiva Do Usuário Sobre O Acompanhamento E O Uso De Psicotrópicos Na Atenção Básica. Essentia - Revista de Cultura, Ciência e Tecnologia Da UVA, 20(2). https://doi.org/10.36977/ercct.v20i2.281

Veras, R. P., \& Oliveira, M. (2018). Envelhecer no Brasil: A construção de um modelo de cuidado. Ciência \& Saúde Coletiva, 23(6), 1929-1936. https://doi.org/10.1590/1413-81232018236.04722018

Wenceslau, L. D., \& Ortega, F. (2015). Saúde mental na atenção primária e Saúde Mental Global: perspectivas internacionais e cenário brasileiro. Interface Comunicação, Saúde, Educação, 19(55), 1121-1132. https://doi.org/10.1590/1807-57622014.1152

Yu, N. W., Chen, P. J., Tsai, H. J., Huang, C. W., Chiu, Y. W., Tsay, W. I., Hsu, J., \& Chang, C. M. (2017). Association of benzodiazepine and Z-drug use with the risk of hospitalisation for fall-related injuries among older people: A nationwide nested case-control study in Taiwan. BMC Geriatrics, 17(1), 1-9. https://doi.org/10.1186/s12877-017-0530-4 